\title{
Regulation of pyruvate kinase isozyme M2 is mediated by the ubiquitin-specific protease 20
}

\author{
SO-RA KIM ${ }^{1}$, JIN-OCK KIM ${ }^{1}$, KEY-HWAN LIM ${ }^{1}$, JI-HYUN YUN $^{1}$, INBO HAN $^{2}$ and KWANG-HYUN BAEK ${ }^{1}$ \\ Departments of ${ }^{1}$ Biomedical Science, ${ }^{2}$ Neurosurgery, CHA University, \\ Bundang CHA General Hospital, Gyeonggi-Do 463-400, Republic of Korea
}

Received November 21, 2014; Accepted January 16, 2015

DOI: $10.3892 /$ ijo.2015.2901

\begin{abstract}
USP20, one of deubiquitinating enzymes (DUBs) belonging to the subfamily of ubiquitin-specific protease (USP), regulates ubiquitin-mediated protein degradation. So far, USP20 has been identified as a binding protein and a regulator of hypoxia-inducible factor (HIF)- $1 \alpha, \beta$-adrenergic receptor, and tumor necrosis factor (TNF) receptor associated factor 6 (TRAF6). In order to investigate other biological functions of USP20 with its novel substrates, we searched for putative substrates through two-dimensional electrophoresis (2-DE) and matrix-assisted laser desorption-ionization timeof-flight mass spectrometry (MALDI-TOF/MS) analysis. We found several putative substrates, some of which are related to cancer metabolism or neural disorders. Among these, the pyruvate kinase isoenzyme M2 (PKM2) had a high identity score. Most cancer cells contain a specific metabolic pathway, referred to as the Warburg effect. One well-known function of PKM2 is a main regulator in cancer metabolic pathways, and PKM2 promotes the Warburg effect and tumor growth. In addition, both PKM2 and HIF-1 $\alpha$ upregulate the expression of target genes. From this evidence, it is expected that USP20 would be associated with the metabolic pathway through the regulation of PKM2 ubiquitination. Despite various roles of DUBs, the biological functions of USP20 in cellular mechanisms are poorly understood. Herein, we investigated the interaction between PKM2 and USP20. Our results suggest a new molecular pathway in cancer metabolism through the regulation of PKM2.
\end{abstract}

\section{Introduction}

Ubiquitination is an important post-translational modification in cells, maintaining homeostasis through numerous cellular

Correspondence to: Professor Kwang-Hyun Baek, Department of Biomedical Science, CHA University, 335 Pangyo-Ro, Bundang-Gu, Seongnam-Si, Gyeonggi-Do 463-400, Republic of Korea

E-mail:baek@cha.ac.kr

Key words: cancer, deubiquitination, MALDI-TOF, ubiquitination processes such as cell growth, proliferation, apoptosis, DNA damage response, and immune response $(1,2)$. Ubiquitin is composed of 76 amino acids and is well conserved in the eukaryotic genome. In addition, ubiquitin regulates its targeted proteins for proteasomal degradation or signal transduction in an ATP-dependent manner through making a covalent bond of lysine sites on its binding proteins (3). Within the cells, polyubiquitination has several different roles according to the position of the attachment site on target proteins (4). Ubiquitin has seven lysine residues (K6, $\mathrm{K} 11$, K27, K29, K33, K48, and K63). On these residues, other ubiquitins are attached as mono- or polyubiquitin chain (1). K48-linked polyubiquitin chains regulate the proteasomal degradation of target proteins (5), and K63-linked and linear polyubiquitin chains control protein kinase activation and cell signaling $(6,7)$.

Deubiquitination is a reversible reaction of ubiquitination and this process requires for deubiquitinating enzymes (DUBs). DUBs can induce deconjugation of the ubiquitin from target proteins and lead to regulation of stability or downstream signaling for targeting proteins. Deubiquitination, in common with ubiquitination, regulates diverse biochemical pathways in cells. The importance of many DUBs has been verified in the receptor tyrosine kinase signaling, signal transduction, gene transcription, DNA repair, proliferation, and mitosis (8). The human genome encodes 100 DUBs; in general, DUBs are divided into at least six subfamilies, as follows: ubiquitin-specific processing proteases (USPs/UBPs), ubiquitin carboxy-terminal hydrolases (UCHs), Jad1/Pad/MPN-domain-containing metallo-enzymes (JAMMs), Otu-domain ubiquitin-aldehydebinding proteins (OTUs), Ataxin-3/Josephin, and monocyte chemotactic protein-induced proteases (MCPIPs) (9-12). These six subfamilies are classified in accordance with the ubiquitinbinding domain. They have specific regions including Cys, Asp/Asn and His boxes in the domain of catalytic activity, and JAMM subfamily members are zinc-dependent metalloproteases (13).

The pyruvate kinase isoform M2 (PKM2) is regarded as a major protein regulating metabolism signaling in cancer. In normal conditions, PKM2 acts as a pyruvate kinase with a tetramer form. In cancer cells, however, PKM2 plays a role as a protein kinase with a dimer form. Expression of the PKM2 dimer impedes the glycolytic metabolism, thereby inducing the Warburg effect. This effect is the main process through 
which most cancer cells predominantly produce energy, involving a high rate of glycolysis (14). Increased conversion of glucose to regulate lactate is a key feature of many cancer cells with rapid growth. Increased PKM2 expression enables regulation of lactate production in cancer cells. In hypoxic condition, PKM2 leads to the accumulation of upstream glycolytic metabolites, which stimulate the pentose phosphate pathway, thereby promoting macromolecule biosynthesis and inhibiting intracellular reactive oxygen species (ROS) generation (15). In addition, PKM2 dimers are translocated into the nucleus and stimulate the transcription factors such as hypoxia-inducible factor (HIF)-1, HIF-2, signal transducer and activator of transcription 4 (STAT4), and octamer-binding transcription factor 4 (Oct-4). Therefore, cell proliferation and cell growth are increased (16).

USP20 is one of the DUBs belonging to the USP subfamily. It has been identified as a regulator of HIF-1 $\alpha$ (17), tumor necrosis factor (TNF) receptor-associated factor 6 (TRAF6), and Tax (18). A recent study suggested that USP20 is also involved in homologous recombination (HR), one of the DNA repair pathways, by stabilizing Rad17 as a DNA damage response protein (19). A previous study revealed that the overexpression of USP20 increased the expression level of HIF-1 $\alpha$ by regulating degradation signal (17). Moreover, PKM2 stimulates the binding of HIF- $1 \alpha$ at the promoter of target genes such as the recruitment of coactivators, histone acetylation, and gene transcription (20). The expression of HIF-1 $\alpha$ is increased in the majority of human cancers and acts as a major regulator of $\mathrm{O}_{2}$ homeostasis (21). In this study, we identified PKM2 as a putative substrate of USP20 with proteomic analysis tools. We also demonstrated that PKM2 can be a binding partner for USP20, which downregulates the ubiquitination level by DUB activity.

\section{Material and methods}

Cell culture and transfection. HeLa cells were cultured in Dulbecco's modified Eagle's medium (Gibco BRL, Rockville, MD, USA) contained with $10 \%$ fetal bovine serum (Gibco BRL) and $1 \%$ penicillin/streptomycin (Gibco BRL). Cells were transfected using $10 \mathrm{mM}$ polyethlylenimine (PEI, Polysciences, Warrington, WA, USA) with $6 \mu$ g plasmid DNA in $100-\mathrm{mm}$ culture dish.

Two-dimensional electrophoresis (2-DE) and matrix assisted laser desorption/ionization time-of-flight mass spectrometry (MALDI-TOF/MS). 2-DE was conducted as previously described (22). Cells were lysed with 2-DE lysis buffer (7 M $\mathrm{CO}\left(\mathrm{NH}_{2}\right)_{2}, 2 \mathrm{MSC}\left(\mathrm{NH}_{2}\right)_{2}, 4.5 \%$ CHAPS, $100 \mathrm{mM}$ DTE, $40 \mathrm{mM}$ Tris, $\mathrm{pH} 8.8$ ), and samples were applied to $\mathrm{pH}$ 3-10 nonlinear gradient strips (Amersham Biosciences, Uppsala, Sweden). IEF was performed at $80,000 \mathrm{Vh}$. After IFR, strips were loaded on 9-16\% linear gradient polyacrylamide gels and the gels were stained with Coomassie Brilliant Blue (CBB) G-250 for $12 \mathrm{~h}$. Stained gels were scanned with a Bio-Rad GS710 densitometer (Bio-Rad Laboratories, Richmond, CA, USA) and analyzed using Image Master Platinum 5.0 image analysis program (Amersham Biosciences, Glattbrugg, Switzerland).

For MALDI-TOF/MS analysis, each peptide was concentrated by a POROS R2, Oligo R3 column (Applied Biosystems,
Foster City, CA, USA). After washing the column with $70 \%$ $\mathrm{CH}_{3} \mathrm{CN}, 100 \% \mathrm{CH}_{3} \mathrm{CN}$ and then $50 \mathrm{mM}\left(\mathrm{NH}_{4}\right) \mathrm{HCO}_{3}$, samples were applied to the R2, R3 column and eluted with cyano4-hydroxycinamic acid (CHCA) (Sigma-Aldrich, St. Louis, MO, USA). Samples were dissolved in $70 \% \mathrm{CH}_{3} \mathrm{CN}$ and $2 \%$ $\mathrm{HCO}_{2} \mathrm{H}$ onto the MALDI plate (Opti-TOF ${ }^{\mathrm{TM}} 384$-well Insert, Applied Biosystems) (23).

MALDI-TOF/MS was performed on 4800 MALDI-TOF/ TOF $^{\mathrm{TM}}$ Analyzer (Applied Biosystems) equipped with a $355-\mathrm{nm}$ Nd:YAG laser. The pressure in the TOF analyzer is approximately 7.6e-07 Torr. The mass spectra were obtained in the reflection mode with an accelerating voltage of $20 \mathrm{kV}$ and sum from either 500 laser pulses and calibrated using the 4700 calibration mixture (Applied Biosystems). NCBInr database (downloaded May 12, 2014, \# of entries = 7,051,549 sequence) searching was performed with the MASCOT search engine (http://www.matrixscience.com). The peak listgenerating software Data Explorer version 4.4 (PerSeptive Biosystems, Wiesbaden, Germany) was used. Database search criteria were, taxonomy, Homo sapiens, fixed modification; carboxyamidomethylated $(+57)$ at cysteine residue; variable modification; oxidized $(+16)$ at methionine residues, maximum allowed missed cleavage, 1 , MS tolerance, $100 \mathrm{ppm}$. Only peptides resulting from trypsin digests were considered, and trypsin peak $(842.5090,2211.1040)$ was used for internal calibration.

Reverse transcription PCR (RT-PCR). Total RNAs were extracted from HeLa cells after $24 \mathrm{~h}$ of transfection using TRIzol $^{\mathrm{TM}}$ reagent (Invitrogen, Carlsbad, CA, USA). cDNA of each sample was constructed by the SuperScript III system (Invitrogen). PCR conditions were $20 \mathrm{sec}$ denaturation at $95^{\circ} \mathrm{C}$, $30 \mathrm{sec}$ annealing at $55^{\circ} \mathrm{C}$, and $40 \mathrm{sec}$ extension at $72^{\circ} \mathrm{C}$ for all genes. All primer sets were assessed by gel electrophoresis to confirm amplification from extracted cDNA of a single band of the expected size.

Immunoprecipitation. After $24 \mathrm{~h}$ of incubation from transfection, cells were harvested. Phosphate buffered saline (PBS) was used as a washing solution. Harvested cells were lysed with a lysis buffer $(150 \mathrm{mM} \mathrm{NaCl}, 1 \%$ Triton X-100, $20 \mathrm{mM}$ Tris- $\mathrm{HCl}$ at $\mathrm{pH} 8.0,1 \mathrm{mM}$ EDTA, $100 \mathrm{mM}$ PMSF, protease inhibitor cocktail). A $1.5 \mathrm{mg}$ aliquot of cell lysates was incubated with mouse anti-Flag (Sigma-Aldrich), mouse anti-PKM2, and mouse anti-Myc (Santa Cruz Biotechnology, Santa Cruz, CA, USA) antibodies at $4^{\circ} \mathrm{C}$. Protein A/G PLUS-Agarose beads (Santa Cruz Biotechnology) was added to the lysates and incubated at $4^{\circ} \mathrm{C}$. Immunoprecipitated proteins were separated on $8 \%$ polyacrylamide gels.

Western blotting. The protein concentration was identified using the Bio-Rad Protein Assay reagent (Bio-Rad Laboratories, Hercules, CA, USA). Cell lysates were boiled with 2X SDS sample buffer and loaded on $8 \%$ polyacrylamide gels. The separated proteins on SDS-PAGE gel were transferred to polyvinylidene difluoride (PVDF) membranes. After transfer to the membranes, they were incubated in $3 \%$ skim milk and primary antibody mixture (1:1000) overnight at $4^{\circ} \mathrm{C}$. Membranes were incubated with the secondary peroxidase labeled anti-mouse IgG antibody (1:10000) (Santa Cruz 
Table I. Proteins identified by MALDI-TOF/MS.

\begin{tabular}{|c|c|c|c|c|c|c|c|}
\hline Place & $\begin{array}{l}\text { Spot } \\
\text { ID }\end{array}$ & $\begin{array}{l}\text { Accession } \\
\text { number }\end{array}$ & Protein name & Score & $\begin{array}{l}\text { Matched peptides } \\
\text { number }\end{array}$ & $\begin{array}{c}\text { Sequence } \\
\text { coverage (\%) }\end{array}$ & $\begin{array}{l}\text { Theoretical } \\
\text { MW/PI }\end{array}$ \\
\hline \multirow[t]{7}{*}{ Myc } & 1538 & gil48928058 & $\begin{array}{l}\text { Small ubiquitin-related } \\
\text { modifier } 3 \text { (SUMO3) precursor }\end{array}$ & 70 & $7 / 50(14 \%)$ & 46 & $11630 / 5.32$ \\
\hline & 1551 & gil5410451 & Interferon-induced protein $\mathrm{p} 78$ & 118 & $18 / 60(30 \%)$ & 37 & $75886 / 5.60$ \\
\hline & 1577 & gil3063997 & $\begin{array}{l}\text { CMP-N-acetylneuraminate- } \beta \text { - } \\
\text { galactosamide- } \alpha-2,3- \\
\text { sialyltransferase } \\
\text { (ST3GAL1) }\end{array}$ & 92 & $11 / 60(18 \%)$ & 41 & $30462 / 9.50$ \\
\hline & 1752 & gil348239 & Unnamed & 78 & $15 / 68(22 \%)$ & 34 & $58083 / 7.60$ \\
\hline & 1694 & gil228008398 & $\begin{array}{l}\text { Synaptotagmin binding, } \\
\text { cytoplasmic } \\
\text { RNA interacting protein } \\
\text { isoform } 3 \text { (SYNCRIP) }\end{array}$ & 65 & $11 / 60(18 \%)$ & 30 & $58927 / 7.18$ \\
\hline & 1696 & gil12804225 & Unknown & 117 & $21 / 60(35 \%)$ & 42 & $59887 / 5.45$ \\
\hline & 2680 & gil16306550 & $\begin{array}{l}\text { Selenium-binding } \\
\text { protein } 1 \text { (SELENBP1) }\end{array}$ & 73 & $10 / 40(25 \%)$ & 33 & $52358 / 5.93$ \\
\hline \multirow[t]{4}{*}{ USP20 } & 1642 & gil193788722 & Hypothetical protein LOC51507 & 67 & $10 / 56(17 \%)$ & 45 & $33865 / 8.86$ \\
\hline & 1731 & gil33286418 & $\begin{array}{l}\text { Pyruvate kinase isozymes } \\
\text { M1/M2 isoform M2 (PKM2) }\end{array}$ & 119 & $18 / 60(30 \%)$ & 34 & $58470 / 7.96$ \\
\hline & 1796 & gil1208427 & ER-60 protease & 117 & $16 / 60(26 \%)$ & 35 & $57160 / 5.98$ \\
\hline & 2608 & gil111550164 & $\begin{array}{l}\text { BORIS transcription factor } \\
\text { transcript variant A5 (CTCFL) }\end{array}$ & 76 & $14 / 43(32 \%)$ & 27 & $71158 / 8.51$ \\
\hline
\end{tabular}

Score is $-10 * \log (\mathrm{P})$, where $\mathrm{P}$ is the probability that the observed match is a random event; it is based on NCBInr database using MASCOT searching program as MS/MS data.

Biotechnology). Then, the bands were detected using X-ray developer and exposure to film.

Immunofluorescence. At $24 \mathrm{~h}$ after transfection, cells were seeded onto 12-mm flame sterilized coverslips. After incubation, cells were fixed with $4 \%$ paraformaldehyde (Sigma-Aldrich). The cells were incubated with primary goat anti-USP20 and mouse anti-PKM2 antibodies (Santa Cruz Biotechnology). Then, the cells were incubated with 1:200 diluted fluoresceinisothiocyanate-labeled anti-goat or anti-mouse $\operatorname{IgG}$ (Molecular Probes, Carlsbad, CA, USA) as a secondary antibody. Cells were stained with 4' 6-diamindino-2-phenylindole (DAPI; Molecular Probes) for nuclei staining. Afterwards, coverslips were mounted onto glass slides using $90 \%$ glycerol/100 mM Tris ( $\mathrm{pH}$ 8.0) and cell images were captured by a confocal microscope (Leica TCS SP8, Leica, Wetzlar, Germany).

DNA constructs and mutagenesis. A catalytically inactive form of USP20 was constructed by site-directed mutagenesis using QuikChange $^{\mathrm{TM}}$ Site-Directed Mutagenesis Kit (Statagene, La Jolla, CA, USA). The forward primer (5'-CCT CGG GAA CTC CAG CTA CAT GAA CGC-3') and reverse primer (5'-GCG TTC ATG TAG CTG GAG TTC CCG AGG-3') were used for replacing cysteine to serine at position 154 of USP20. The HA-ubiquitin (K48R) and HA-ubiquitin (K63R) constructs were as previously described (24).

\section{Results}

Investigation of putative substrates for USP20. It has been demonstrated that USP20 is involved in intracellular regulatory mechanisms such as proliferation of cancer cells, DNA repair, and the immune system (17-19). TRAF6, HIF-1 $\alpha$, and Rad17 were identified as USP20 associated proteins. When the USP20 is overexpressed, the expression of TRAF6 and HIF-1 $\alpha$ is stabilized (17-19). USP20 has important roles in tumorigenesis, the immune system, and DNA repair, other additional roles of USP20 are not yet fully understood. In order to investigate other binding partners of USP20, we performed 2-DE and MALDI-TOF/MS analysis using HeLa cells with overexpression of USP20. Protein spots were detected in the acrylamide gel (Fig. 1). Among them, 11 upregulated spots were detected. Before the proteomic analysis, we verified the expression of USP20 in HeLa cells (Fig. 1A), and performed 2-DE analysis to identify putative substrates of USP20 (Fig. 1B). In USP20transfected samples, 7 were downregulated, and 4 were upregulated (Fig. 1C and D). Isolated spots were analyzed by mass spectrometry (Table I).

To investigate whether the screened substrates are regulated by the ubiquitin-proteasome pathway, we performed RT-PCR to determine the effect of USP20 overexpression on the transcription (Fig. 2, Table II). As expected, the results indicate that USP20 has no effect on the transcriptional level 


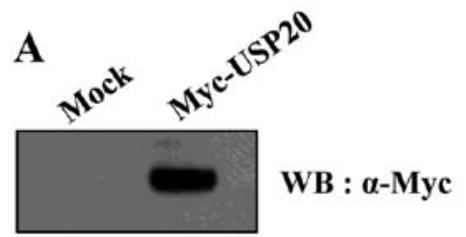

B

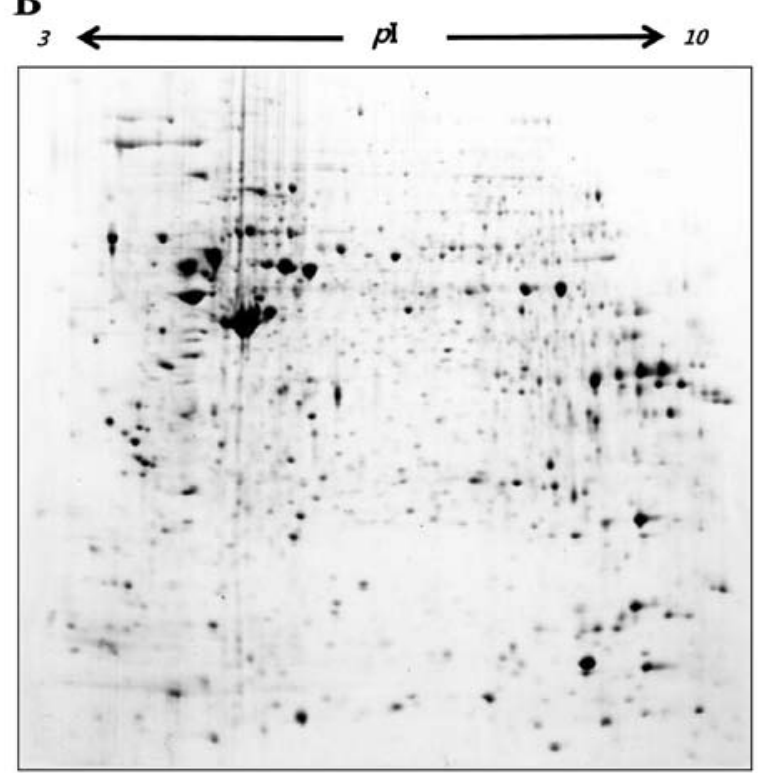

Mock

C
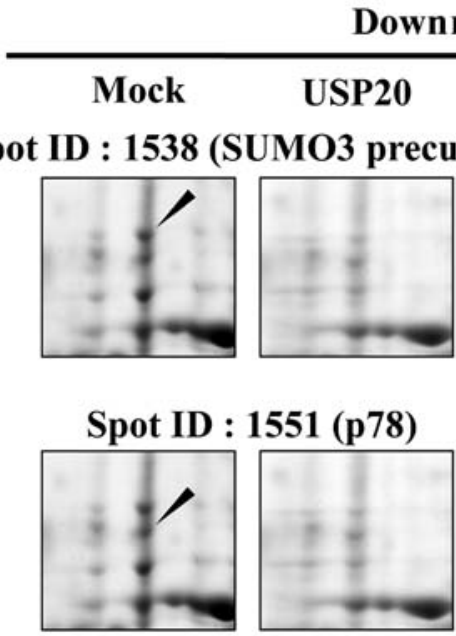

$1551(p 78)$

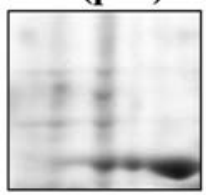

Spot ID : 1577 (ST3GAL1)
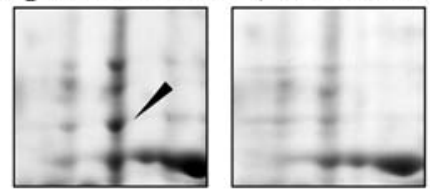

Spot ID : 1694 (SYNCRIP)
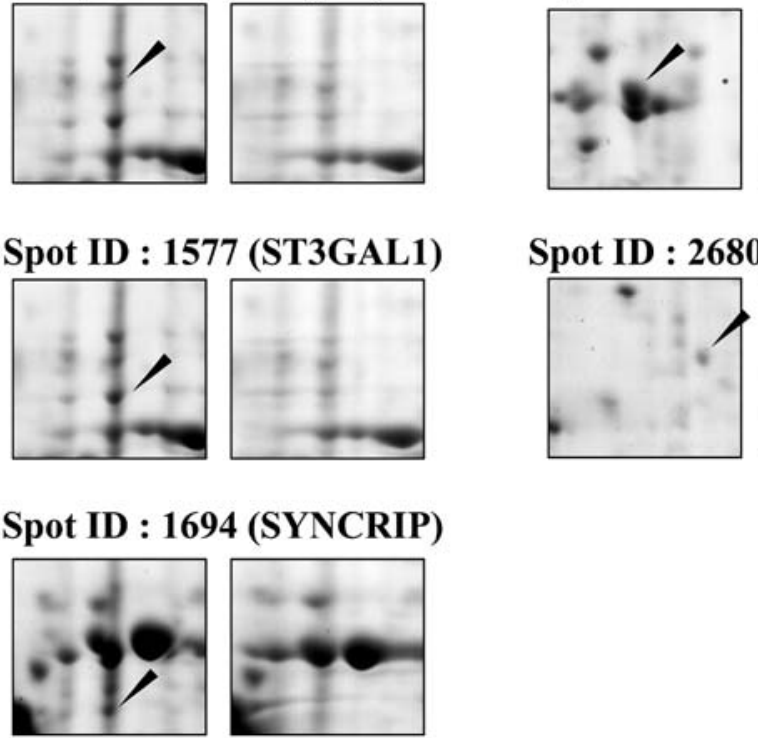
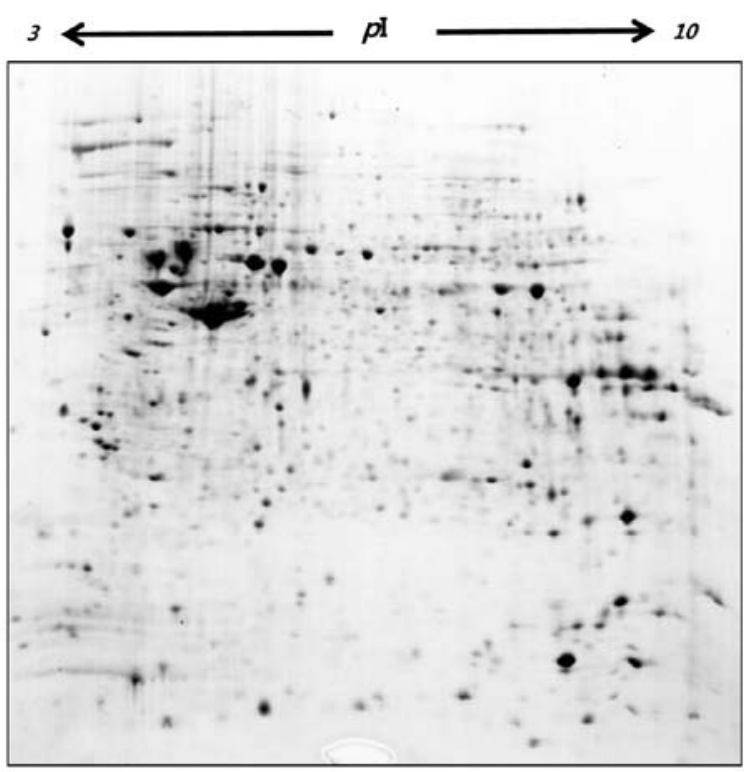

Myc-USP20

D

Upregulation

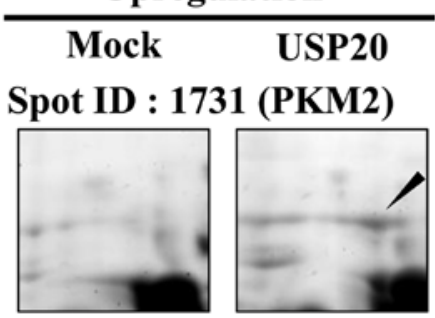

Spot ID : 1796 (ER-60 protease)
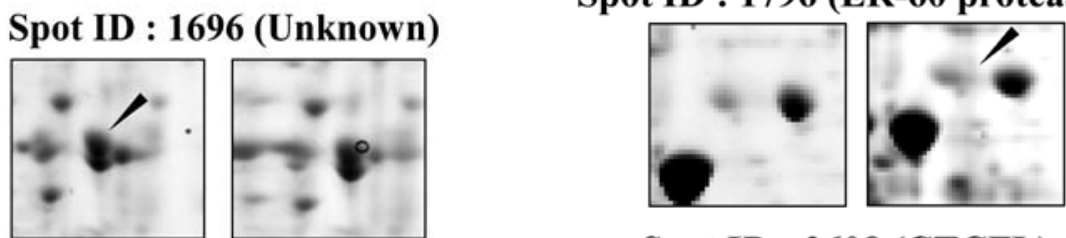

Spot ID : 2608 (CTCFL)

Spot ID : 2680 (SELENBP1)
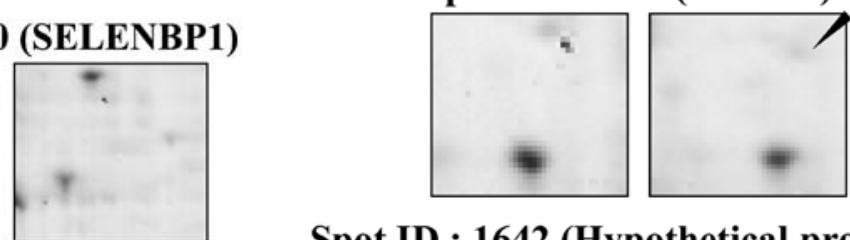

Spot ID : 1642 (Hypothetical protein)

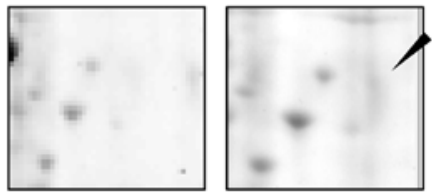

Figure 1. Investigation of putative substrates for USP20. (A) Expression of Myc-USP20 for 2-DE analysis. A mock control is a $p c D N A-6 m y c$ empty vector. (B) Result of 2-DE analysis. pcDNA-6myc-Usp20 or pcDNA3-3Myc was transfected into HeLa cells. Samples were loaded onto the polyacrylamide gel. (C and D) Difference in spot size on the 2-DE gel. Eleven proteins were identified, and down- and up-regulated spots appeared under USP20 overexpression. 
Table II. Primers used for RT-PCR analysis.

Name

Sequences

Small ubiquitin-related modifier 3 precursor

Interferon-induced protein $\mathrm{p} 78$

CMP-N-acetylneuraminate- $\beta$-galactosamide- $\alpha-2,3$-sialyltransferase

Unnamed

Synaptotagmin binding, cytoplasmic RNA interacting protein isoform 3

Unknown

Selenium-binding protein 1

Hypothetical protein LOC51507

Pyruvate kinase isozymes M1/M2 isoform M2

ER-60 protease

BORIS transcription factor transcript variant A5
FP: 5'-CCA AGG AGG GTG TGA AGA CA-3' RP: 5'-GTG TCC TCG TCC TCC ATC TC-3'

FP: 5'-GGG AAG GAA TGG GAA TCA GT-3' RP: 5'-ATG CTG AGA GCC TCT GTG GT-3'

FP: 5'-GGG AGA TGC CAT CAA CAA GT-3' RP: 5'-TGT TTC CAG AAA CCC TTT CG-3'

FP: 5'-ATA TGC CAC TCC GTG GAA AG-3' RP: 5'-GAA GGA GCC TTC ACT GCA TC-3'

FP: 5'-TGT GGG AAA GAT CCC AAG AG-3' RP: 5'-TTG GCA ACT GAG ATG CAG AC-3' FP: 5'-GAT GGC TGA GAT TGC TGT GA-3' RP: 5'-TTG GTT TGG GTG GTT CAA AT-3' FP: 5'-ATC ACC GAC ATC CTG CTC TC-3' RP: 5'-CCG TTT TCC CTT GAC CAC TA-3'

FP: 5'-GAG AGC GAA GCT GGA AAA GA-3' RP: 5'-ACT GTC AGC GAT GGA CCT CT-3'

FP: 5'-ATG AGT ACC ATG CGG AGA CC-3' RP: 5'-AGT CCA GCC ACA GGA TGT TC-3' FP: 5'-ATG GGC CTG TGA AGG TAG TG-3' RP: 5'-GTG GCA TCC ATC TTG GCT AT-3' FP: 5'-CTA CAA GCT GAA ACG CCA CA-3' RP: 5'-AGC AGA ACA GTA GCG GCA TT-3' FP: 5'-ACC ACA GTC CAT GCC ATC AC-3' RP: 5'-TCC ACC ACC CTG TTG CTG TA-3 of genes encoding proteins identified from proteomics analysis (Fig. 2). From the data, we selected one of the proteins, pyruvate kinase isozymes M1/M2 isoform M2 (PKM2) for further analysis. A previous study showed that PKM2 functions as a co-activator with HIF-1 $\alpha$ (21). In addition, it has been demonstrated that HIF-1 $\alpha$ is stabilized by USP20 (17). Our proteomic analysis with USP20 overexpression showed that PKM2 has a high identity score and exhibits two times higher expression than that of a control. Furthermore, the mRNA level was not different in the USP20-transfected condition (Fig. 2B). Collectively, these results indicate that PKM2 can be a genuine protein for one of the UPS20 substrates.

USP20 interacts with PKM2. To demonstrate the binding between USP20 and PKM2, we performed immunoprecipitation analysis using transfected samples with $M y c$ - or Flag-tagged USP20 and PKM2 expressing HeLa cells. Interaction of these two proteins was confirmed by immunoprecipitation assay using an anti-Myc or an antiFlag antibody. As expected, the result showed that USP20 binds with PKM2 exogenously (Fig. 3A). We next performed immunofluorescence (IF) assay using HeLa cells to check the localization of these two proteins in HeLa cells (Fig. 3B). The results showed that both USP20 and PKM2 were co-localized in the cytosol and nucleus. Taken together, our biochemical assay revealed that USP20 was specifically bound with PKM2.
PKM2 is regulated by the ubiquitin-proteasome system. A previous study revealed the posttranslational modification of PKM2 (25). For example, extracellular signal-regulated kinase (ERK) phosphorylates PKM2, leading to the induction of c-Myc expression as a transcription factor with $\beta$-catenin as a co-transcription factor in the nucleus (25). However, evidence of PKM2 that undergoes the proteasomal degradation pathway has not yet been found. Since we demonstrated the interaction between USP20 and PKM2 (Fig. 3), we next determined whether PKM 2 is degraded by the $26 \mathrm{~S}$ proteasome. The level of ubiquitination for PKM2 was dramatically increased with the treatment of MG132, a proteasome inhibitor (Fig. 4A). Polyubiquitination has different roles in cells depending on the lysine site. While Lys48-linked polyubiquitination is involved in the degradation of proteins through the $26 \mathrm{~S}$ proteasome, Lys63-linked polyubiquitination is related to intracellular signaling pathways (26). In order to analyze the ubiquitination pattern of PKM2, we designed mutant constructs of ubiquitin; HA-ubiquitin (K48R) and HA-ubiquitin (K63R). Polyubiquitination has different roles in cells depending on the lysine site. K48R and K63R constructs were only Lys48 or Lys63 changed to Arg. On transfection of HA-ubiquitin $(K 48 R)$, we found a dramatically increased ubiquitination level of PKM2. In contrast, the K63-mutated ubiquitin did not increase PKM2 ubiquitination (Fig. 4B). These results suggest that PKM2 can build polyubiquitin chains on both K48 and $\mathrm{K} 63$, and be regulated by the ubiquitin proteasome pathway. 

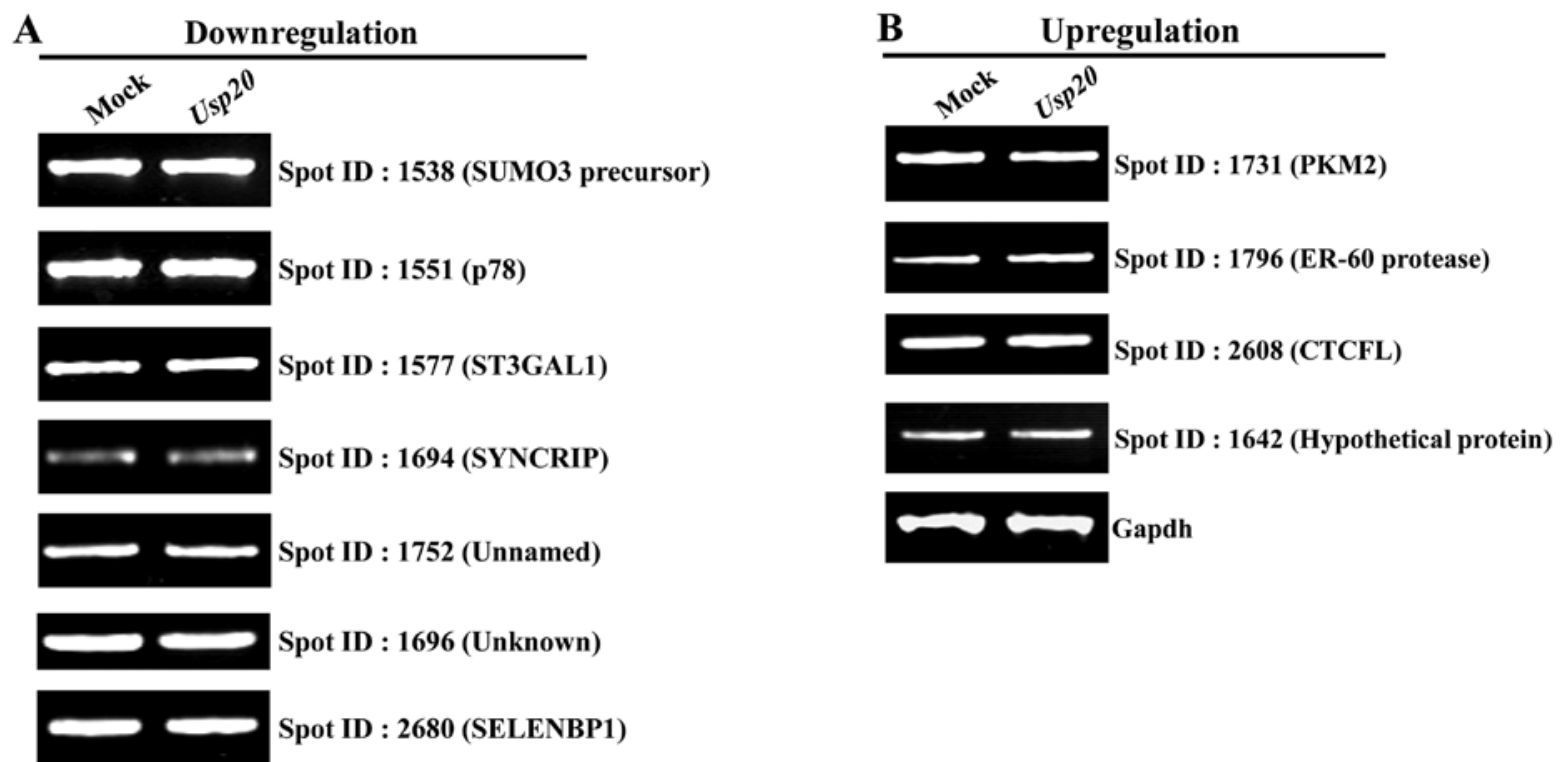

Figure 2. Analysis of mRNA expression levels using RT-PCR. mRNA levels of identified candidates were downregulated (A) or upregulated (B). Gapdh was used as a control for mRNA expression.
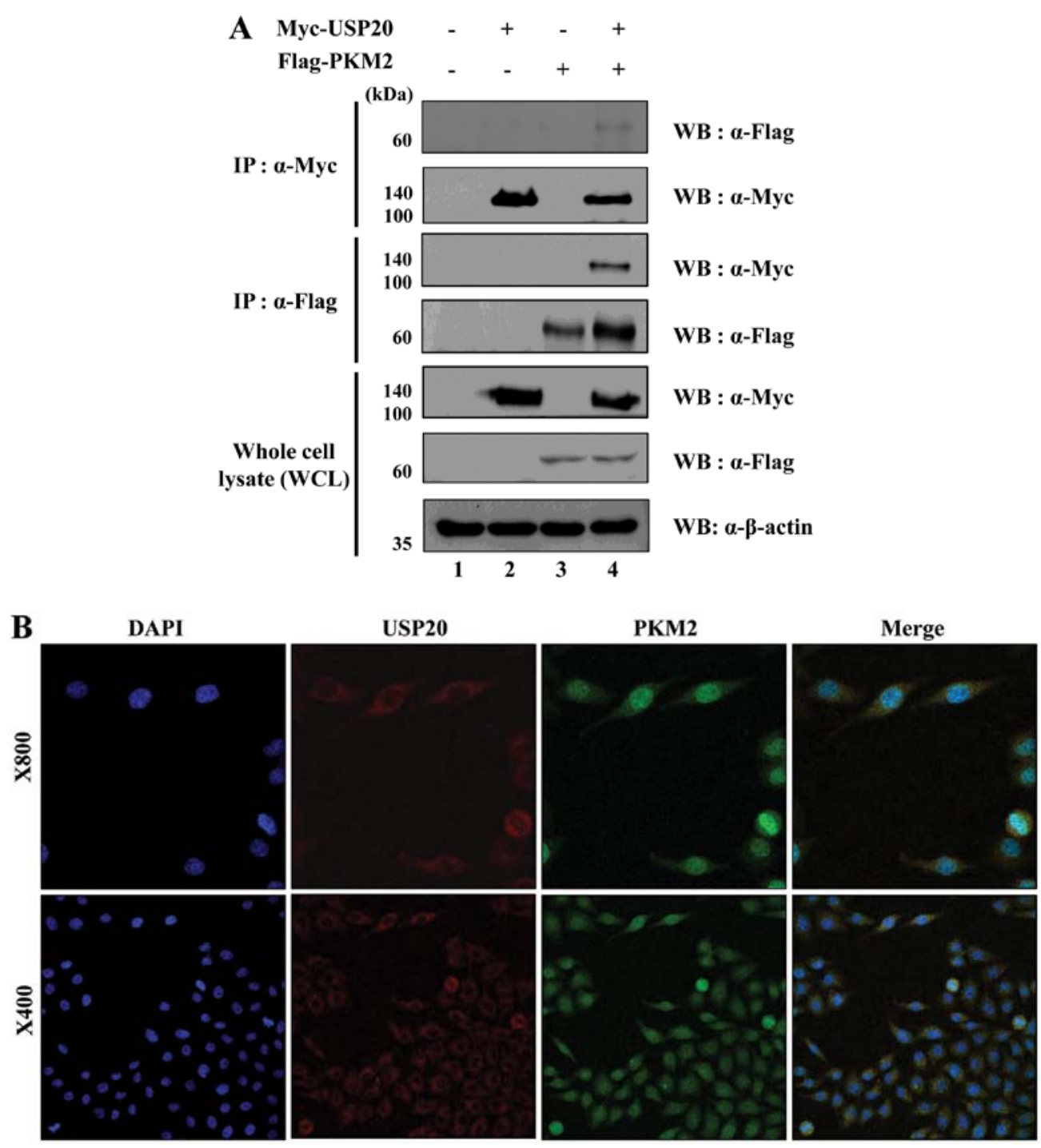

Figure 3. USP20 binds with PKM2 (A) HeLa cells were transfected with pcDNA-3Myc-Usp20 and pCS4-3Flag-Pkm2 plasmids. Anti-Flag and anti-Myc antibodies were used for co-immunoprecipitation. (B) USP20 and PKM2 were detected with an anti-USP20 (red) or an anti-PKM2 (green) antibody. The nuclei were stained using DAPI (4',6'-diamidino-2-phenylindole) (blue). 


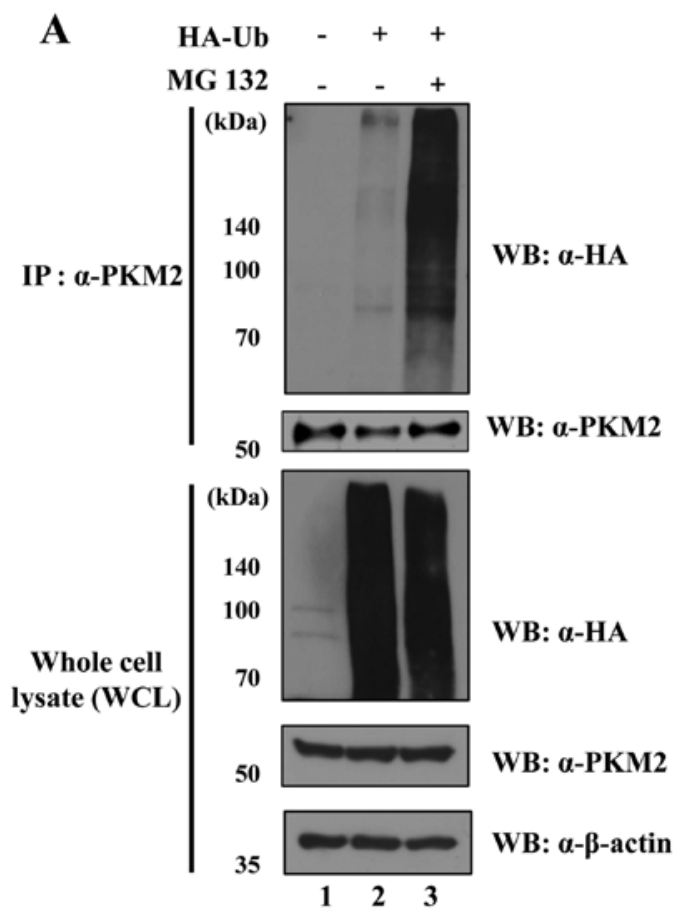

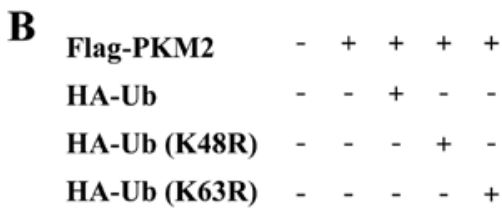

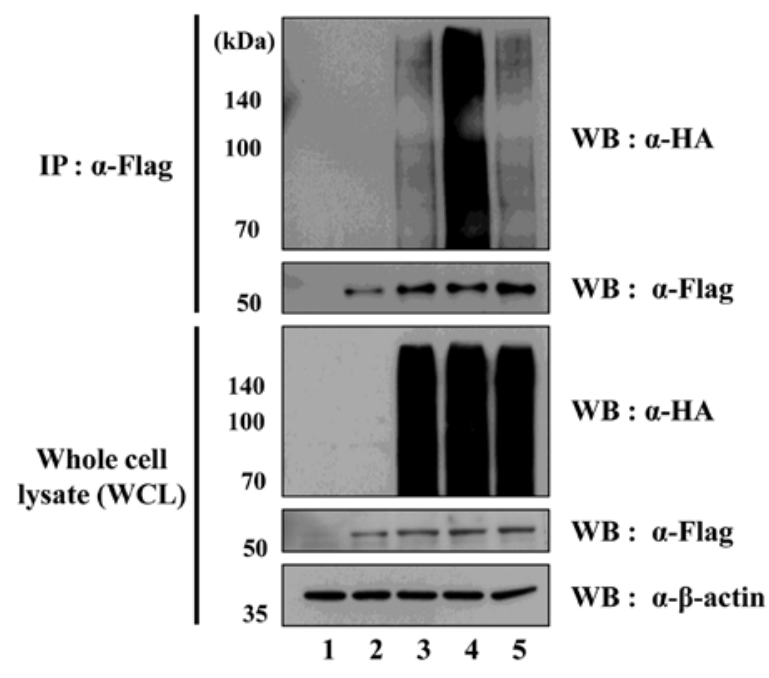

Figure 4. Ubiquitination of PKM2. (A) HA-ubiquitin was transfected into HeLa cells. Immunoprecipitation was performed using an anti-PKM2 antibody. MG132 was used as a proteasomal inhibitor. (B) HA-ubiquitin (K48R) and pCS4-3Flag-Pkm2 or HA-ubiquitin (K63R) and pCS4-3Flag-Pkm2 were co-transfected into HeLa cells. All the samples were treated with MG132.

USP20 regulates $P K M 2$ by its DUB activity. To identify the molecular mechanism by which USP20 engages in DUB activity for PKM2 regulation, Usp2O and $P k m 2$ were expressed with HA-ubiquitin in HeLa cells. Additionally, USP20 (C153S) lacking catalytic activity was used as a negative control for catalytic activity for USP20. When Flag-PKM2 was immunoprecipitated by an anti-Flag antibody, the wild-type USP20 dramatically reduced the ubiquitination level of PKM2. However, the catalytically inactive form of USP20 and USP12 as a negative control could not affect the ubiquitination level of PKM2 (Fig. 5). These data confirm that USP20 is a specific DUB for PKM2.

To determine whether USP20 has effect on the regulation of PKM2 by DUB activity, we expressed USP20 protein in a dose-dependent manner in HeLa cells. The result indicated that PKM2 expression did not increase in the USP20 transfected condition (Fig. 5C). Taken together, these data indicate that USP20 can be a specific DUB for PKM2, and it would regulate the signaling progress of PKM2.

\section{Discussion}

Ubiquitination is a crucial mechanism for regulating a number of cellular processes (27). DUBs mediate the reversible reaction of ubiquitination by depolymerization and removal of ubiquitin from target proteins. These enzymes are very important in cellular homeostasis and cancer $(28,29)$. Many studies have demonstrated the importance of DUBs and their functions in cancers and other cellular functions. In the mechanism of carcinogenesis, DUBs not only play a tumor suppression role in cancer cells, but also have oncogenic function by stabilizing some oncogenes (30). Therefore, specific inhibitors of DUBs have been proposed as putative therapeutic targets (31).
Herein, we investigated USP20, as an important enzyme in diverse cellular mechanisms, regulating TRAF6, HIF1- $\alpha$, $\beta_{2} \mathrm{AR}$, and Rad17 $(18,32)$. However, the detailed mechanisms of USP20 activity, and its diverse functions are not fully understood compared to the other DUBs.

In this study, we performed 2-DE analysis and identified PKM2 as one of the target proteins for USP20. PKM2 is a main protein in tumor metabolism. In 1924, Otto Warburg identified a specific metabolism in cancer cells. In cancer cells, glucose was converted to lactate in the normoxia, that is known as the Warburg effect, or aerobic glycolysis (33). In this effect, PKM2 accumulates pyruvate converting it to lactate. As a result, the pentose phosphate pathway (PPP) is promoted and macromolecules are synthesized for use in the proliferation of cancer cells (34). When PKM2 is localized in the nucleus, it interacts with HIF- $1 \alpha$ and promotes the transactivation of encoding cell proliferation proteins in cancer cells. In the prolyl hydroxylase 3 (PHD3)-stimulated condition, PHD3 enhances the binding with PKM2 and HIF-1 $\alpha$. The binding of PKM2 and HIF-1 $\alpha$ induces the expression of HIF-1 $\alpha$-targeted proteins, such as GLUT1, LDHA, PDK1, and VEGF, GLUT1, LDHA, and PDK1 inducing the glycolytic pathway in cancer cells, while VEGF is an angiogenesis protein (20).

We confirmed the interaction between USP20 and PKM2, using a co-immunoprecipitation assay and immunofluorescence analysis, and the result showed that USP20 was bound with PKM2 and they are co-localized in the cytosol (Fig. 3). We also investigated the patterns of ubiquitination for PKM2. We generated mutant constructs of ubiquitin, and found that PKM2 is ubiquitinated by Lys48- and Lys63-polyubiquitin chains.

In summary, we found PKM2 to be a target of USP20 and demonstrated the specific deubiquitination of PKM2 by USP20. Through these results, it is feasible to elucidate 

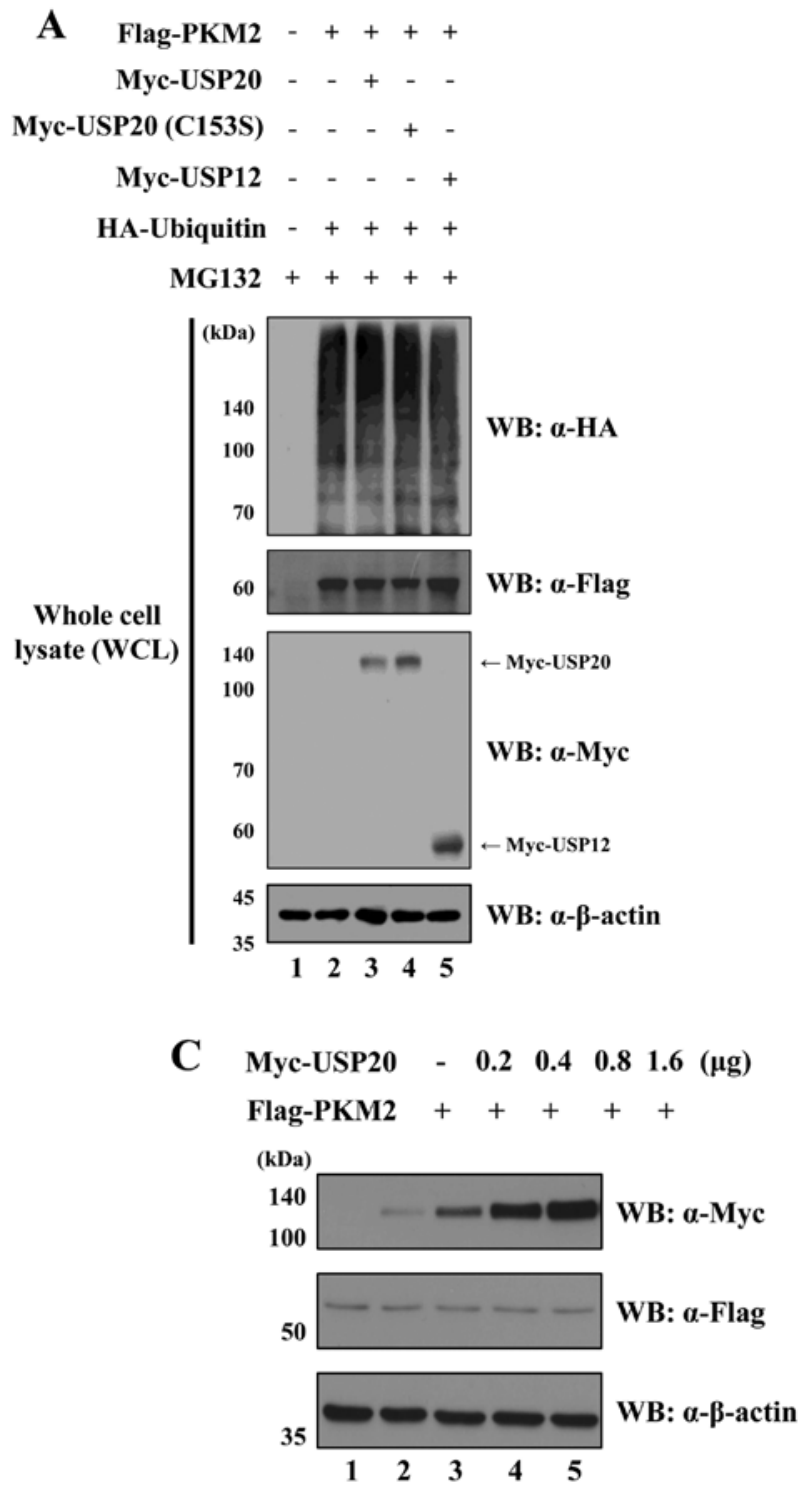
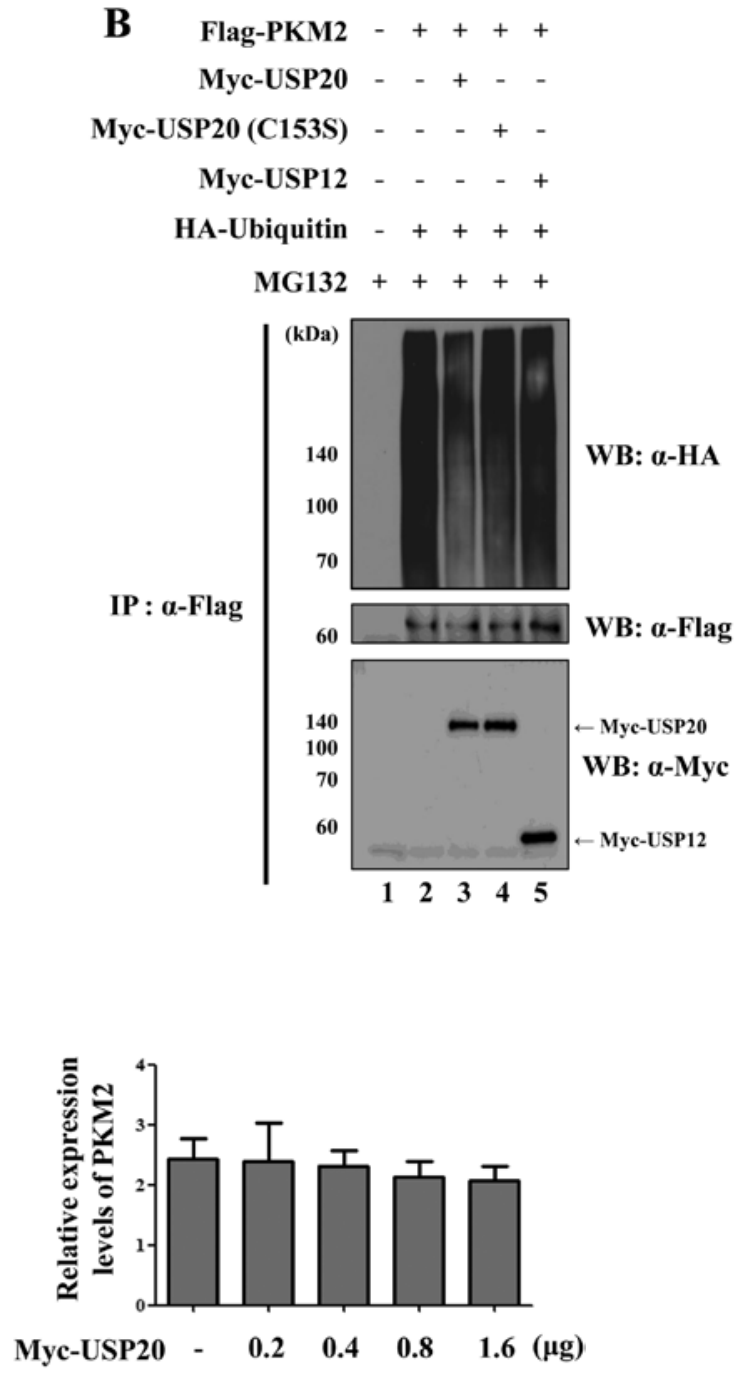

Figure 5. Deubiquitination of PKM2. (A and B) pcDNA-6myc-Usp20 or pcDNA-6myc-Usp20 (C154S) were transfected into HeLa cells. All the samples were treated with MG132. USP12 was used as a negative control. (C) pcDNA-6myc-Usp20 was transfected into HeLa cells in a dose-dependent manner and pCS4-Flag-Pkm2 was transfected with pcDNA-6myc-Usp20.

the cellular cancer mechanism and identify a new target for cancer therapy. In order to investigate the diverse regulation of USP20 and PKM2, further functional studies should be performed.

\section{Acknowledgements}

This work was supported by Basic Science Research Program through the National Research Foundation of Korea (NRF) funded by the Ministry of Education, Science and Technology (2013-0141), and by Exchange Professor Program of LG Yonam Foundation (2012).

\section{References}

1. Yang WL, Zhang $X$ and Lin HK: Emerging role of Lys-63 ubiquitination in protein kinase and phosphatase activation and cancer development. Oncogene 29: 4493-4503, 2010.

2. Cole AJ, Clifton-Bligh RJ and Marsh DJ: Ubiquitination and cancer: Histone H2B monoubiquitination: roles to play in human malignancy. Endocr Relat Cancer 22: T19-T33, 2015.
3. Tu Y, Chen C, Pan J, Xu J, Zhou ZG and Wang CY: The Ubiquitin Proteasome Pathway (UPP) in the regulation of cell cycle control and DNA damage repair and its implication in tumorigenesis. Int J Clin Exp Pathol 5: 726-738, 2012.

4. Weissman AM, Shabek N and Ciechanover A: The predator becomes the prey: Regulating the ubiquitin system by ubiquitylation and degradation. Nat Rev Mol Cell Biol 12: 605-620, 2011.

5. Hershko A and Ciechanover A: The ubiquitin system for protein degradation. Annu Rev Biochem 61: 761-807, 1992.

6. Kawadler H and Yang X: Lys63-linked polyubiquitin chains: Linking more than just ubiquitin. Cancer Biol Ther 5: 1273-1274, 2006.

7. Rieser E, Cordier SM and Walczak H: Linear ubiquitination: A newly discovered regulator of cell signalling. Trends Biochem Sci 38: 94-102, 2013.

8. Hussain S, Zhang Y and Galardy PJ: DUBs and cancer: The role of deubiquitinating enzymes as oncogenes, non-oncogenes and tumor suppressors. Cell Cycle 8: 1688-1697, 2009.

9. Todi SV and Paulson HL: Balancing act: Deubiquitinating enzymes in the nervous system. Trends Neurosci $34: 370-382$, 2011.

10. Lim KH, Ramakrishna S and Baek KH: Molecular mechanisms and functions of cytokine-inducible deubiquitinating enzymes. Cytokine Growth Factor Rev 24: 427-431, 2013. 
11. Kouranti I, McLean JR, Feoktistova A, Liang P, Johnson AE, Roberts-Galbraith RH and Gould KL: A global census of fission yeast deubiquitinating enzyme localization and interaction networks reveals distinct compartmentalization profiles and overlapping functions in endocytosis and polarity. PLoS Biol 8: e1000471, 2010.

12. Bhattacharya $\mathrm{S}$ and Ghosh MK: Cell death and deubiquitinases: perspectives in cancer. Biomed Res Int 2014: 435197, 2014.

13. MacGurn JA, Hsu PC and Emr SD: Ubiquitin and membrane protein turnover: From cradle to grave. Annu Rev Biochem 81: 231-259, 2012.

14. Ferreira LM: Cancer metabolism: The Warburg effect today. Exp Mol Pathol 89: 372-380, 2010.

15. Grüning NM, Rinnerthaler M, Bluemlein K, Mülleder M, Wamelink MM, Lehrach H, Jakobs $C$, Breitenbach $M$ and Ralser M: Pyruvate kinase triggers a metabolic feedback loop that controls redox metabolism in respiring cells. Cell Metab 14: 415-427, 2011.

16. Gao X, Wang H, Yang JJ, Liu X and Liu ZR: Pyruvate kinase M2 regulates gene transcription by acting as a protein kinase. Mol Cell 45: 598-609, 2012.

17. Li Z, Wang D, Messing EM and Wu G: VHL protein-interacting deubiquitinating enzyme 2 deubiquitinates and stabilizes HIF-1alpha. EMBO Rep 6: 373-378, 2005.

18. Yasunaga J, Lin FC, Lu X and Jeang KT: Ubiquitin-specific peptidase 20 targets TRAF6 and human T cell leukemia virus type 1 tax to negatively regulate NF-kappaB signaling. J Virol 85: 6212-6219, 2011.

19. Shanmugam I, Abbas M, Ayoub F, Mirabal S, Bsaili M, Caulder EK, Weinstock DM, Tomkinson AE, Hromas R and Shaheen M: Ubiquitin-specific peptidase 20 regulates Rad17 stability, checkpoint kinase 1 phosphorylation and DNA repair by homologous recombination. J Biol Chem 289: 22739-22748, 2014.

20. Luo W, Hu H, Chang R, Zhong J, Knabel M, O'Meally R, Cole RN, Pandey A and Semenza GL: Pyruvate kinase M2 is a PHD3-stimulated coactivator for hypoxia-inducible factor 1. Cell 145: 732-744, 2011.

21. Semenza GL: Regulation of metabolism by hypoxia-inducible factor 1. Cold Spring Harb Symp Quant Biol 76: 347-353, 2011.

22. Kim YS, Kim MS, Lee SH, Choi BC, Lim JM, Cha KY and Baek KH: Proteomic analysis of recurrent spontaneous abortion: Identification of an inadequately expressed set of proteins in human follicular fluid. Proteomics 6: 3445-3454, 2006.
23. Choi BK, Cho YM, Bae SH, Zoubaulis CC and Paik YK: Single-step perfusion chromatography with a throughput potential for enhanced peptide detection by matrix-assisted laser desorption/ ionization-mass spectrometry. Proteomics 3: 1955-1961, 2003.

24. Ramakrishna S, Suresh B, Lee EJ, Lee HJ, Ahn WS and Baek KH: Lys-63-specific deubiquitination of SDS3 by USP17 regulates HDAC activity. J Biol Chem 286: 10505-10514, 2011.

25. Yang W, Zheng Y, Xia Y, Ji H, Chen X, Guo F, Lyssiotis CA, Aldape K, Cantley LC and Lu Z: ERK1/2-dependent phosphorylation and nuclear translocation of PKM2 promotes the Warburg effect. Nat Cell Biol 14: 1295-1304, 2012.

26. Perrett CA, Lin DY and Zhou D: Interactions of bacterial proteins with host eukaryotic ubiquitin pathways. Front Microbiol 2: 143 , 2011.

27. Ramakrishna S, Suresh B and Baek KH: The role of deubiquitinating enzymes in apoptosis. Cell Mol Life Sci 68: 15-26, 2011.

28. D'Arcy P, Brnjic S, Olofsson MH, et al: Inhibition of proteasome deubiquitinating activity as a new cancer therapy. Nat Med 17: 1636-1640, 2011.

29. Vucic D, Dixit VM and Wertz IE: Ubiquitylation in apoptosis: A post-translational modification at the edge of life and death. Nat Rev Mol Cell Biol 12: 439-452, 2011.

30. Fraile JM, Quesada V, Rodríguez D, Freije JM and López-Otín C: Deubiquitinases in cancer: New functions and therapeutic options. Oncogene 31: 2373-2388, 2012.

31. Mattern MR, Wu J and Nicholson B: Ubiquitin-based anticancer therapy: Carpet bombing with proteasome inhibitors vs surgical strikes with E1, E2, E3, or DUB inhibitors. Biochim Biophys Acta 1823: 2014-2021, 2012.

32. Berthouze M, Venkataramanan V, Li Y and Shenoy SK: The deubiquitinases USP33 and USP20 coordinate beta2 adrenergic receptor recycling and resensitization. EMBO J 28: 1684-1696, 2009.

33. Warburg O: On the origin of cancer cells. Science 123: 309-314, 1956.

34. Semenza GL: HIF-1: Upstream and downstream of cancer metabolism. Curr Opin Genet Dev 20: 51-56, 2010. 